\title{
Ebola Virus Disease in the Democratic Republic of Congo
}

\author{
Gaël D. Maganga, D.V.M., Ph.D., Jimmy Kapetshi, M.D., \\ Nicolas Berthet, Pharm.D., Ph.D., Benoît Kebela Ilunga, M.D., \\ Felix Kabange M.D., Placide Mbala Kingebeni, M.D., Vital Mondonge, M.D., \\ Jean-Jacques T. Muyembe, M.D., Ph.D., Eric Bertherat, M.D., Sylvie Briand, M.D., \\ Joseph Cabore, M.D., Alain Epelboin, M.D., Pierre Formenty, D.V.M., M.P.H., \\ Gary Kobinger, M.D., Licé González-Angulo, M.Sc., Ingrid Labouba, Ph.D., \\ Jean-Claude Manuguerra, Ph.D., Jean-Marie Okwo-Bele, M.D., \\ Christopher Dye, D. Phil., and Eric M. Leroy, D.V.M., Ph.D.
}

ABSTRACT
BACKGROUND

The seventh reported outbreak of Ebola virus disease (EVD) in the equatorial African country of the Democratic Republic of Congo (DRC) began on July 26, 2014, as another large EVD epidemic continued to spread in West Africa. Simultaneous reports of EVD in equatorial and West Africa raised the question of whether the two outbreaks were linked.

\section{METHODS}

We obtained data from patients in the DRC, using the standard World Health Organization clinical-investigation form for viral hemorrhagic fevers. Patients were classified as having suspected, probable, or confirmed EVD or a non-EVD illness. Blood samples were obtained for polymerase-chain-reaction-based diagnosis, viral isolation, sequencing, and phylogenetic analysis.

\section{RESULTS}

The outbreak began in Inkanamongo village in the vicinity of Boende town in Équateur province and has been confined to that province. A total of 69 suspected, probable, or confirmed cases were reported between July 26 and October 7, 2014, including 8 cases among health care workers, with 49 deaths. As of October 7 , there have been approximately six generations of cases of EVD since the outbreak began. The reported weekly case incidence peaked in the weeks of August 17 and 24 and has since fallen sharply. Genome sequencing revealed Ebola virus (EBOV, Zaire species) as the cause of this outbreak. A coding-complete genome sequence of EBOV that was isolated during this outbreak showed $99.2 \%$ identity with the most closely related variant from the 1995 outbreak in Kikwit in the DRC and 96.8\% identity to EBOV variants that are currently circulating in West Africa.

\section{CONCLUSIONS}

The current EVD outbreak in the DRC has clinical and epidemiologic characteristics that are similar to those of previous EVD outbreaks in equatorial Africa. The causal agent is a local EBOV variant, and this outbreak has a zoonotic origin different from that in the 2014 epidemic in West Africa. (Funded by the Centre International de Recherches Médicales de Franceville and others.)
From the Centre International de Recherches Médicales de Franceville, World Health Organization (WHO) Collaborating Center, Franceville, Gabon (G.D.M., N.B., I.L., E.M.L.); Ministry of Health (B.K.I., F.K.), Institut National de Recherche Biomédicale (J.K., P.M.K., J.J.T.M.), and the WHO (V.M., J.C.), Kinshasa, Democratic Republic of Congo; Centre National de la Recherche Scientifique, Unité Epidémiologie et Physiopathologie des Virus Oncogènes (CNRS UMR3569) (N.B.), Eco-anthropologie et Ethnobiologie, UMR 7206 CNRS-MNHN (A.E.), and Institut Pasteur, Unité de Recherche et d'Expertise Environnement et Risques Infectieux, Cellule d'Intervention Biologique d'Urgence (J.-C.M.), Paris, and Institut de Recherche pour le Développement, Unité Maladies Infectieuses et Vecteurs Ecologie, Genetique, Evolution et Controle IRD 224-CNRS 5290-UM1-UM2, Montpellier (E.M.L.) - all in France; Public Health Agency of Canada, Winnipeg (G.K.); and the WHO, Geneva (E.B., S.B., P.F., L.G.-A., J.-M.O.-B., C.D.). Address reprint requests to Dr. Dye at the WHO, 20 Ave. Appia, CH-1211 Geneva, Switzerland, or at dyec@ who.int.

Drs. Maganga, Kapetshi, and Berthet contributed equally to this article.

This article was published on October 15 , 2014, and updated on October 21, 2014, at NEJM.org.

N EnglJ Med 2014;371:2083-91. DOI: 10.1056/NEJMoal411099 Copyright (@) 2014 Massachusetts Medical Society. 
N AUGUST 24, 2014, WHEN THE EYES OF the world were on the spreading West African outbreak of Ebola virus disease (EVD), the World Health Organization (WHO) was notified of another EVD outbreak in the vicinity of Boende town, Équateur province, in western Democratic Republic of Congo (DRC). Boende town lies $700 \mathrm{~km}$ northeast of the capital city, Kinshasa, and $300 \mathrm{~km}$ east of Mbandaka, the capital of Équateur province (Fig. 1). The affected area is situated in humid tropical forest and delimited by two large rivers, which are the main channels for moving people and goods to and from Mbandaka. The roads in the area of Boende town are in poor condition, as are the bridges that cross a multitude of smaller rivers. There are few commercial flights in and out of Mbandaka (two to three each week) and none out of Boende.

This is the seventh Ebola outbreak in the DRC since the first was reported in $1976 .{ }^{1}$ The index patient was a pregnant woman living in Inkanamongo village (Watsi Kengo Health Area) who butchered a monkey (of an unknown arboreal species) that had been found dead by her husband. She became ill on July 26 and died on August 11. A local doctor and three health workers who undertook a postmortem cesarean section (to separate the fetus from the mother before burial, according to the local culture) also became infected and died. These health workers were evidently the source of further cases in this outbreak.

We report the cases and deaths that have been identified as of October 7, on the geographic distribution and rate of spread of infection, and on the identity of the agent causing this EVD outbreak, a novel Ebola virus (EBOV, Zaire species) variant, in comparison with variants isolated from West Africa.

\section{METHODS}

\section{CASE DEFINITIONS}

Epidemiologic and clinical data were recorded on the standard WHO clinical-investigation form for viral hemorrhagic fevers ${ }^{2}$ with the use of standard case definitions, which are provided in the Supplementary Appendix (available with the full text of this article at NEJM.org; also see video, available at NEJM.org). A suspected case (either before or after death) was defined as a sudden onset of high fever in a patient who had contact with a person with suspected, probable, or confirmed EVD, with a dead or sick animal, or with any person with a sudden onset of high fever and at least three of the following symptoms or clinical signs: headache, vomiting, anorexia, diarrhea, lethargy, stomach pain, aching muscles or joints, difficulty swallowing or breathing, or hiccup; unexplained bleeding; or any sudden, unexplained death. A probable case was defined as any suspected case evaluated by a clinician or any person who died from suspected EVD and had an epidemiologic link to a confirmed case but was not tested and did not have laboratory confirmation of the disease. A probable or suspected case was classified as confirmed when a sample from the affected person tested positive for EBOV in the laboratory. In this study, the final classification of patients remained as suspected or probable when a definitive diagnosis could not be made (usually because no blood sample was obtained). We attempted to identify the source of infection in each patient with EVD by tracing contacts, mainly retrospectively.

\section{BLOOD SAM PLES}

The first samples were collected from eight symptomatic patients with suspected EVD who visited the Lokolia health center in Équateur province. As in previous EVD outbreaks, blood samples were collected, with oral consent, either at the homes of patients or in hospital isolation wards, by a team that included staff members of the Ministry of Health in the DRC and the WHO. ${ }^{3}$ Samples were placed in dry tubes and immediately transported to Institut National de Recherche Biomédicale (INRB), Kinshasa, DRC, for laboratory testing and storage. Blood samples were also sent to the WHO reference center for the diagnosis of viral hemorrhagic fever, the Centre International de Recherches Médicales de Franceville (CIRMF), Gabon, for confirmation. At all stages, essential biosecurity measures were taken to avoid contamination of personnel and the environment.

\section{DIAGNOSTIC ASSAYS}

Total RNA was extracted from $100 \mu \mathrm{l}$ of serum with the use of BioRobot EZ1 and the EZ1 Virus Mini Kit, version 2.0 (both from Qiagen), according to the manufacturer's instructions. RNA was first converted to complementary DNA (cDNA) with the use of a commercial kit (High Capacity cDNA Reverse Transcription Kit, Applied Biosystems), and specific real-time reverse-transcriptasepolymerase-chain-reaction (RT-PCR) assays targeting the nucleoprotein gene of $\mathrm{EBOV}^{4}$ were 


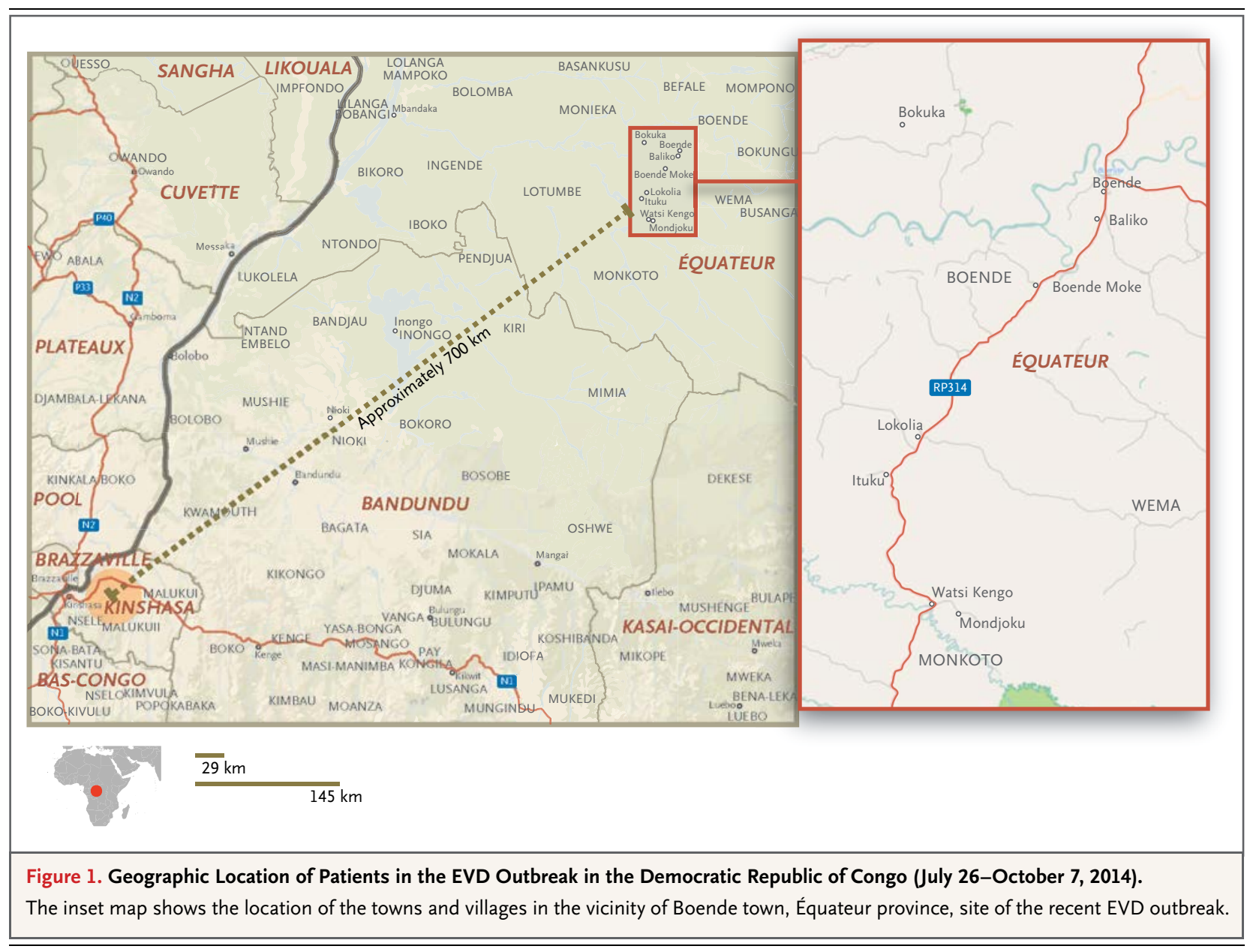

performed on the 7500 Fast Real-Time PCR System (Applied Biosystems). The amplification cycle involved 2 minutes at $55^{\circ} \mathrm{C}$ and an initial denaturation at $95^{\circ} \mathrm{C}$ for 10 minutes, followed by 45 cycles at $95^{\circ} \mathrm{C}$ for 15 seconds and at $58^{\circ} \mathrm{C}$ for 1 minute.

For samples with positive results on RT-PCR, a PCR assay targeting a fragment of the filovirus polymerase (L) gene was performed with the use of the SuperScript III One-Step RT-PCR Kit (Invitrogen). The reaction was carried out in a final volume of $25 \mu \mathrm{l}$, containing $12.5 \mu \mathrm{l}$ of $2 \mathrm{X}$ buffer, $1.5 \mu \mathrm{l}$ of each primer (10 $\mu \mathrm{M})$ (Filo A and Filo B), bovine serum albumin ( $1 \mu \mathrm{g}$ per microliter), $1 \mu \mathrm{l}$ of SuperScript III Platinum Taq Mix, RNase-free water, and $5 \mu \mathrm{l}$ of RNA. Amplification involved a step of reverse transcription for 30 minutes at $45^{\circ} \mathrm{C}$, which was followed by denaturation at $95^{\circ} \mathrm{C}$ for 2 minutes and then by 45 cycles of 15 seconds at $94^{\circ} \mathrm{C}, 30$ seconds at $55^{\circ} \mathrm{C}$, and 45 seconds at $68^{\circ} \mathrm{C}$, with a final elongation step of 5 minutes at $68^{\circ} \mathrm{C}$.

\section{VIRAL SEQUENCING}

The PCR fragment of $346 \mathrm{bp}$ was purified by ultrafiltration before sequencing (Millipore). Sequencing was performed with the use of the BigDye Terminator v1.1 Cycle Sequencing Kit (Applied Biosystems) and purified by means of ethanol precipitation. Sequence chromatograms from both strands were obtained on an automated sequence analyzer (ABI 3730XL, Applied Biosystems) with the PCR primers. For coding-complete genomic characterization, viral RNA was directly extracted from serum samples with the EZ1 Viral RNA Kit (Qiagen), according to the manufacturer's instructions. Extracted total RNA was retrotranscribed into cDNA with SuperScript III reverse transcriptase and random hexamer primers (Life Technologies). The cDNA that was generated was amplified with Phi29 polymerase, as described previously. ${ }^{5}$ Amplified DNA was quantified with the use of the QuantiT DNA Assay Kit (Life Technologies), and a di- 
lution was performed in order to obtain $0.2 \mathrm{ng}$ of amplified DNA per microliter in the final solution. The library was performed with the use of the Nextera XT DNA Sample Preparation Kit (Illumina), according to the manufacturer's instructions.

DNA was initially sequenced with the MiSeq sequencer (Illumina), following by a second run with the Illumina NextSeq sequencer. A total of $5.6 \times 10^{6}$ reads were obtained for each sample. All reads were filtered according to quality, and those corresponding to human genomes were removed with mapping software with the use of the Homo sapiens hg19 sequence as reference. The viral reads corresponding to the EBOV genome were selected by means of a similarity approach with the use of the BLASTN search tool, referring to the complete sequence of a 1976 Yambuku isolate of EBOV available in GenBank (accession number, KC242801). For each selected read, only the region that matched the viral genome was considered. All reads were assembled with SPAdes (St. Petersburg genome assembler) software in order to obtain the coding-complete viral genome. $^{6}$

\section{PHYLOGENETIC ANALYSIS}

We aligned 29 complete genome sequences of EBOV that are available in GenBank with the new DRC (abbreviated COD) sequence of EBOV (accession number, KM519951), using the ClustalW algorithm and MEGA 5 software package. ${ }^{7}$ We used the Bayesian Markov chain Monte Carlo method in MrBayes, version 3.2, software to infer the phylogenetic trees, using two runs of four chains with 1 million generations, with a burn-in rate of $25 \%$ and the GTR+G+I nucleotide substitution model. ${ }^{8}$

\section{VIRAL ISOLATION}

Tissue cultures were performed in a biosafety level 4 laboratory. Monolayers of Vero cells in sixwell plates were incubated for 1 hour at $37^{\circ} \mathrm{C}$ with $50 \mu \mathrm{l}$ of serum from each sample at 1:10 dilution in Dulbecco's modified Eagle's medium. Medium containing $2.5 \%$ fetal-calf serum and antibiotic mix was added $(5 \mathrm{ml})$, and cells were incubated at $37^{\circ} \mathrm{C}$ in $5 \%$ carbon dioxide for 7 days. Supernatant was harvested and stored at $-80^{\circ} \mathrm{C}$ until it was used for genomic characterization. Viral growth in the cell lines was confirmed with the use of a specific real-time RT-PCR assay, as described above.

\section{RESULTS}

\section{CLINICAL AND EPIDEMIOLOGIC ANALYSIS}

From July 26 to October 7, 2014, we identified 69 patients with EVD that was suspected (3 patients), probable (28 patients), or confirmed (38 patients). Of these patients, 33 were male and 36 were female; $80 \%$ of the patients were adults between the ages of 21 and 60 years (Fig. 2A). Among the patients with suspected disease, a higher proportion of children than adults were found to have non-EVD illnesses. Of the 69 patients, 21 male patients and 28 female patients died, including 3 children under the age of 5 years, resulting in a case fatality rate of $74 \%$ among probable and confirmed cases, if all cases and deaths were reported. For 28 paired contacts of patients with EVD, the median (serial) interval between dates of the reported onset of symptoms was 16 days (range, 3 to 27 ), and the mean ( \pm SD) was $16.1 \pm 4.4$ days, similar to that in West Africa. ${ }^{2}$ For 32 of the 49 patients who died, the median time from reported symptom onset to death was 11 days (range, 1 to 30 ), and the mean was $11.3 \pm 6.8$; the interval was not measured for the other patients who died. All 8 health workers who were affected - 4 with probable EVD and 4 with confirmed EVD - died. As compared with patients with suspected EVD who were found to have non-EVD illnesses, patients with probable or confirmed EVD were more likely to have fever, headache, diarrhea, vomiting or nausea, fatigue, anorexia, muscle pain, difficulty swallowing, conjunctivitis, and blood in stools or vomit (Table 1).

This outbreak has been driven by human-tohuman transmission, but all cases and contacts appear to have originated from a single index case - in other words, there was no evidence of more than one transfer of infection from an animal reservoir. So far, all cases have been confined to Équateur province, including Boende town (approximately 45,000 inhabitants, with four Health Areas affected), Boende Moke, Bokongo, Lokolia, Lokula, Mondombe, and Watsi Kengo (ranging from 4000 to 12,000 inhabitants), which are situated on or close to road RP314 (Fig. 1). The majority of suspected, probable, or confirmed cases of EVD have been reported from Lokolia (39 cases) or Watsi Kengo (18 cases), with only 4 cases reported from Boende town and another 8 from other towns in the district.

The maximum number of cases was reported in the weeks beginning August 17 and 24, accord- 


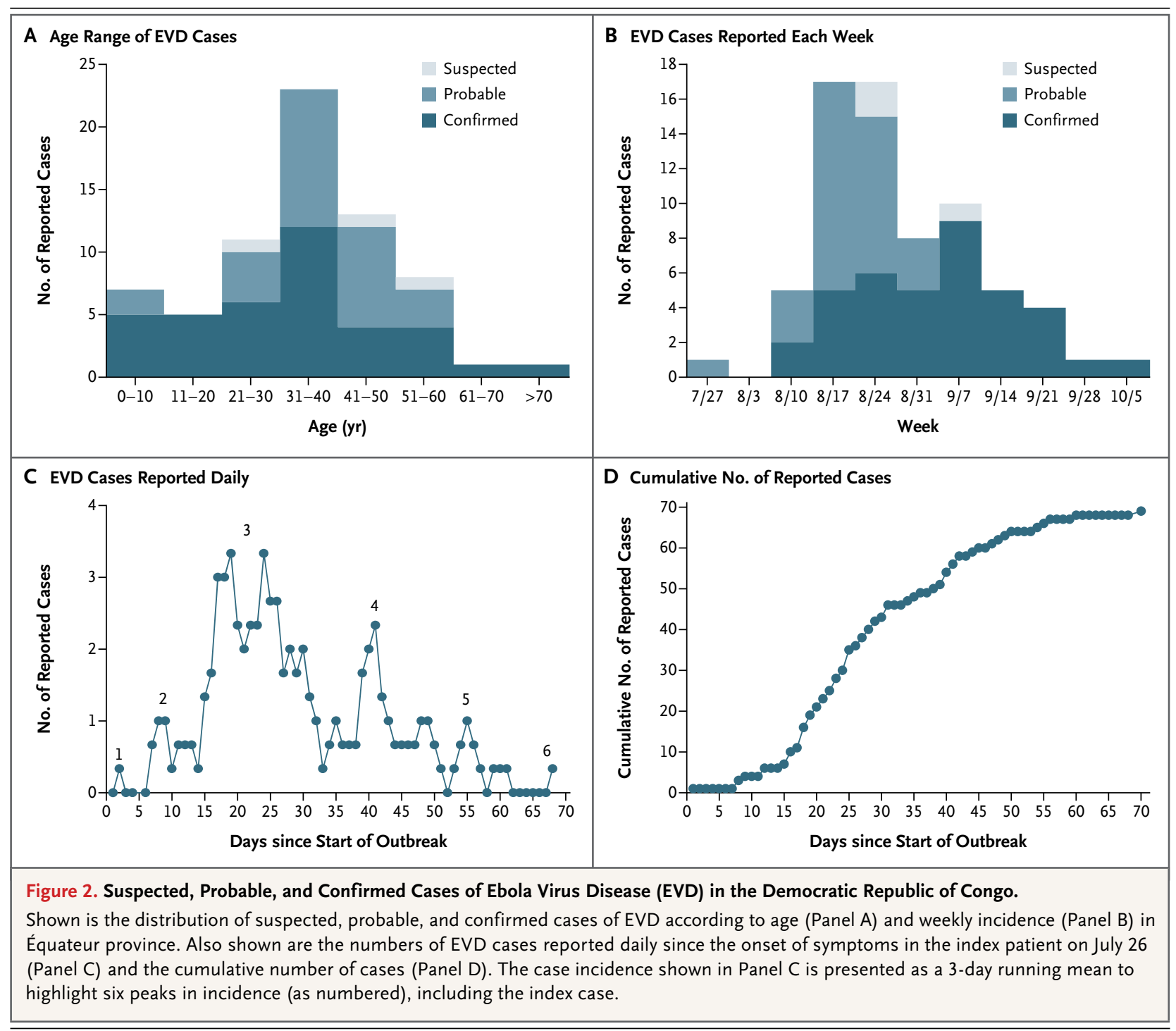

ing to the date of the onset of symptoms (Fig. 2B). It is possible, by plotting the daily case incidence, to discern several generations of cases, as indicated by six peaks in incidence, including the initial index case (Fig. 2C). These covered approximately five serial intervals (average, 16.1 days), generating a total of 69 cases in 70 days (Fig 2D).

The rise in case incidence during August was apparently driven by multiple infections acquired from the index case. Of the 29 patients in whom EVD was diagnosed during first 24 days of the outbreak, 21 were reported to be direct contacts of the index case (i.e., they had physical contact or contact with bodily fluids). If all these secondary cases did indeed acquire infection from a single source, this represents a basic case reproduction number ${ }^{2}\left(R_{0}\right)$ of 21 for this outbreak.
The number of secondary cases arising from each primary case during this outbreak was highly variable. Among other patients with EVD who infected named contacts, 1 patient generated 3 secondary cases, 2 patients generated a further 2 cases each, 30 patients generated a single extra case, and 11 patients generated no further cases. Counting all secondary cases arising among named contacts, including the index case, the average case reproduction number $(R)$ for the whole outbreak was 1.29 (95\% confidence interval [CI], -4.71 to 7.29 ). However, after the exclusion of the 21 cases generated by the index case, the average case reproduction number during the outbreak was 0.84 ( $95 \% \mathrm{CI},-0.38$ to 2.06), which is below the threshold value for persistent transmission $(R>1)$. This explains the 


\begin{tabular}{|c|c|c|c|c|c|}
\hline \multirow[t]{2}{*}{ Sign or Symptom } & $\begin{array}{c}\text { Non- } \\
\text { EVD Cases } \\
(\mathrm{N}=60)\end{array}$ & $\begin{array}{l}\text { Probable } \\
\text { EVD Cases } \\
(\mathrm{N}=\mathbf{2 8 )}\end{array}$ & $\begin{array}{c}\text { Odds Ratio of } \\
\text { Probable EVD } \\
(95 \% \mathrm{Cl}) \dot{\mathrm{i}}\end{array}$ & $\begin{array}{c}\text { Confirmed } \\
\text { EVD Cases } \\
(\mathrm{N}=38)\end{array}$ & $\begin{array}{c}\text { Odds Ratio of } \\
\text { Confirmed EVD } \\
(95 \% \mathrm{Cl}) 广\end{array}$ \\
\hline & \multicolumn{2}{|c|}{ no. of patients (\%) } & \multicolumn{3}{|c|}{ no. of patients (\%) } \\
\hline Fever & $41(68)$ & $28(100)$ & NA & $35(92)$ & 5.4 (1.7 to 9.1$)$ \\
\hline Headache & $7(12)$ & $7(25)$ & 2.5 (-0.7 to 5.7 ) & $17(45)$ & 6.1 (3.4 to 8.9) \\
\hline Diarrhea & $22(37)$ & $23(82)$ & $7.9(4.7$ to 11.1$)$ & $26(68)$ & 3.7 (1.4 to 8.9 ) \\
\hline Abdominal pain & $12(20)$ & $7(25)$ & $1.3(-1.9$ to -4.5$)$ & $18(47)$ & $3.6(1.1$ to 6.1$)$ \\
\hline Vomiting or nausea & $18(30)$ & $22(79)$ & 8.6 (5.4 to 11.8 ) & $26(68)$ & 5.1 (2.6 to 7.5$)$ \\
\hline $\begin{array}{l}\text { Intense fatigue or general } \\
\text { weakness }\end{array}$ & $11(18)$ & $11(39)$ & $2.9(-0.3$ to 6.1$)$ & $27(71)$ & 10.9 (8.3 to 13.5$)$ \\
\hline Anorexia & $9(15)$ & $11(39)$ & 3.7 (0.5 to 6.9$)$ & $15(39)$ & 3.7 (1.1 to 6.3$)$ \\
\hline Muscle pain & $3(5)$ & $7(25)$ & 6.3 (3.1 to 9.5 ) & $17(45)$ & 15.4 (11.6 to 19.1$)$ \\
\hline Difficulty swallowing & $3(5)$ & $6(21)$ & $5.2(2.0$ to 8.4$)$ & $10(26)$ & 6.8 (2.9 to 10.7 ) \\
\hline Difficulty breathing & $5(8)$ & $6(21)$ & $3.0(-0.2$ to 6.2$)$ & $6(16)$ & 2.1 (-1.5 to 5.6$)$ \\
\hline Cough & $5(8)$ & $5(18)$ & $2.4(-0.8$ to 5.6$)$ & $2(5)$ & 0.6 (-4.8 to 6.0$)$ \\
\hline Rash & $1(2)$ & $2(7)$ & 4.5 (1.3 to 7.7$)$ & $3(8)$ & 5.1 ( -4.9 to 15.0$)$ \\
\hline Bleeding from injection site & 0 & $2(7)$ & NA & $3(8)$ & NA \\
\hline Gingivitis & $1(2)$ & $3(11)$ & $7.1(3.9$ to 10.3$)$ & $3(8)$ & $5.1(-4.9$ to 15.0$)$ \\
\hline Conjunctivitis & $1(2)$ & $4(14)$ & 9.8 (6.6 to 13.0$)$ & $6(16)$ & 11.0 (2.4 to 19.7$)$ \\
\hline Bloody or black stools & $4(7)$ & $12(43)$ & 10.5 (7.3 to 13.7$)$ & $8(21)$ & $3.7(0.1$ to 7.3$)$ \\
\hline Vomiting blood & $3(5)$ & $10(36)$ & $10.6(7.4$ to 13.8$)$ & $6(16)$ & $3.6(-0.7$ to 7.8$)$ \\
\hline Bleeding from nose & 0 & $4(14)$ & NA & $4(11)$ & NA \\
\hline Bleeding from vagina & 0 & $2(7)$ & NA & $4(11)$ & NA \\
\hline
\end{tabular}

* EVD denotes Ebola virus disease, and NA not applicable.

$\dagger$ Odds ratios were calculated for patients with probable or confirmed EVD as compared with those who had negative test results for EVD. $\mathrm{Cl}$ denotes confidence interval.

observed decline in the EVD case incidence after mid-August. The last reported patient with EVD became ill on October 4, and no further cases were reported as of October 7.

A total of 1121 contacts of the patients were registered for follow-up. By October 7, a total of 830 had been followed for at least 21 days, which is considered to be the maximum incubation period. The index patient and her contacts had no history of travel to the EVD-affected countries in West Africa (Guinea, Liberia, Nigeria, Senegal, and Sierra Leone) and no history of contact with residents of the affected areas.

\section{IDENTIFICATION AND CHARACTERIZATION OF THE VIRUS}

To identify the causative agent of the Boende EVD outbreak, investigators at Institut National de Recherche Biomédicale in Kinshasa and at Centre International de Recherches Médicales de
Franceville in Gabon analyzed eight samples from patients who were suspected of having EDV. On the basis of both a conventional filoviridaespecific RT-PCR assay targeting a conserved region in the L gene and a real-time RT-PCR assay targeting the nucleoprotein gene in EBOV, six of the eight samples tested positive for EBOV. Sequencing of the 346-bp fragment amplified from the $\mathrm{L}$ gene in four of the samples revealed the viral sequences. These four sequences were identical and closely related to the EBOV variant that caused an EVD outbreak around Kikwit, Zaire (now part of the DRC), in 1995, indicating that the current outbreak in the DRC was unrelated to the ongoing outbreak in West Africa (Fig. 3). We obtained a coding-complete sequence of the virus from the sample having the highest RNA load and obtained a sequence of 18,953 nucleotides in length, which was called EBOV/H.sap/ COD/14/Boe-Lok according to the recently estab- 


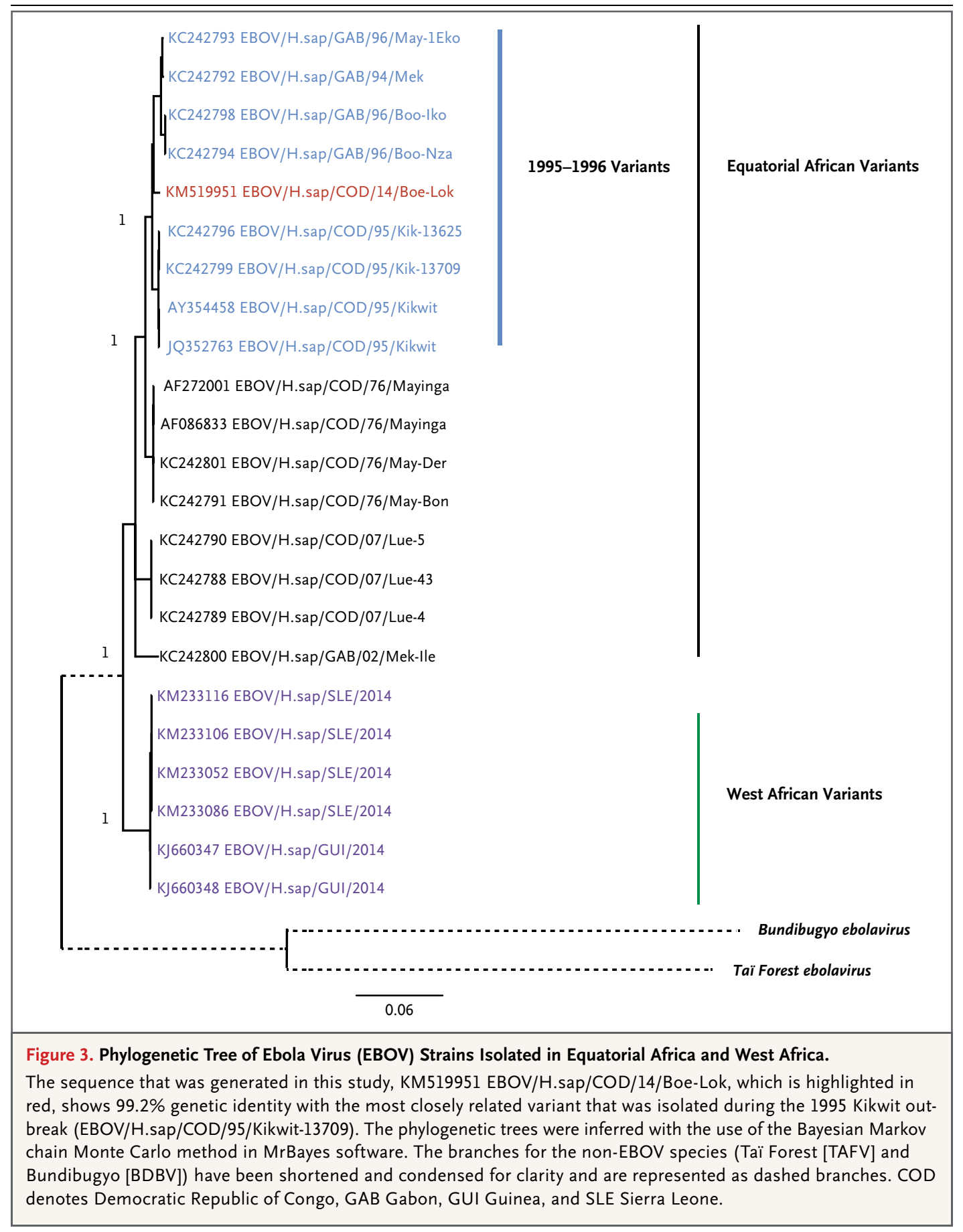

lished filovirus variant nomenclature ${ }^{6}$ (GenBank accession number, KM519951; see also GenBank numbers KM517570.1 and KM517571.1, which were obtained from initial RT-PCR analysis). The sequence showed $99.2 \%$ identity $(0.8 \%$ difference, 145 mutations) with the most closely related variant that was isolated during the 1995 Kikwit outbreak (EBOV/H.sap/COD/95/Kikwit-
13709). Among the 145 mutations, 22 were nonsynonymous, but none were expected to induce any important change in viral protein sequences. In particular, we observed 5 nonsynonymous mutations in the NP gene, 1 in the VP35 gene, 1 in the VP40 gene, 8 in the GP gene, none in the VP30 gene, 2 in the VP24 gene, and 5 in the L gene.

We then performed high-throughput sequenc- 
ing on two additional positive samples after random amplification of extracted total RNA from serum samples. Given the moderate viral load in the serum, we could not obtain the complete sequences but only $2270 \mathrm{bp}$ from one sample and $12,501 \mathrm{bp}$ from the other sample. Analysis of these partial sequences showed an identity of $100 \%$ with the complete sequence obtained previously, further suggesting that the current outbreak in the DRC is due to a single introduction of this novel EBOV variant into the human population.

In contrast to the similarity between the Boende variant and other equatorial African variants (especially EBOV/H.sap/COD/95/Kikwit-13709), the gene sequence of the Lokolia isolate showed only $96.8 \%$ identity (3.2\% difference) with the West African variants, with 601 mutations as compared with a Guinean variant (KJ660347) and 602 mutations as compared with a Sierra Leonean variant (KM233116).

\section{DISCUSSION}

EVD first emerged in human populations in 1976, causing nearly simultaneous, but unrelated, outbreaks in Zaire (now DRC) and Sudan (now South Sudan). The EBOV strain that is causing the present outbreak in the DRC is most closely related to another EBOV variant, which was isolated from a patient in Kikwit (then in Zaire) in 1995. On the basis of the genetic characterization of the virus, together with the geographic location of the outbreak, it is clear that the present outbreak in the DRC is an independent event that has no epidemiologic or virologic connection with the continuing epidemic in West Africa. ${ }^{9}$

The rise and fall of EVD cases in the Boende area between July and October 2014 suggests that the present EVD outbreak in the DRC will probably be typical of other EVD outbreaks in equatorial Africa - that is, characterized by a comparatively low incidence of spillover (probably originating in a local animal reservoir) and chains of human-to-human transmission that are brought under control within 2 to 3 months. Nearly one third of the cases (21 of 69) in this outbreak appear to have arisen from direct contact with the index case. In subsequent chains of transmission, each patient generated relatively few secondary cases, and the average case reproduction number $(R=0.84)$ was below the threshold for persistent transmission (i.e., $R<1$ ).

There are at least five possible reasons why previous EVD outbreaks in equatorial Africa, and the present outbreak in the DRC, have been smaller than the current epidemic in West Africa. The first is that cultural practices associated with EVD differ between equatorial Africa and West Africa, so behaviors and customs in equatorial Africa carry a lower risk of infection among potential contacts. However, customs in the two regions of Africa appear to have much in common - in the way relatives and friends attend the sick and in funeral rites and burial practices, all of which involve bodily contact. In the two regions, there are examples of the use of nonmedical diagnostics and therapies (by herbalists, diviners, healers, and pastors), inadequate availability of disinfectants and protective equipment, and lack of knowledge of microbiologic hygiene by health care workers, along with local resistance to public health measures proposed by national authorities.

The second possible reason why EVD outbreaks have been smaller in equatorial Africa is that infection and illness caused by EBOV variants that are characteristic of equatorial Africa, including the variant that emerged in the Boende district, take a different clinical course from those in West Africa, with different epidemiologic consequences. We have found that equatorial and West African variants of EBOV are genetically distinct, as did Baize et al. ${ }^{9}$ In addition, the genetic diversity among equatorial African isolates appears to be low, as compared with the extensive variation, including many nonsynonymous mutations, that has been identified among West African EBOV isolates, even though the equatorial African variants have been collected over a period of nearly four decades. ${ }^{10}$ Against this background, there is currently no evidence that variants from the two regions are associated with such factors as differences in the infectious period, case fatality rate, or frequency of hemorrhagic disease.

The third possible explanation for the smaller outbreaks in equatorial Africa, such as the one in the Boende area, is that they have largely occurred in remote forested areas, where the number of human contacts is limited in small populations living at low density, with infrequent or slow connections by road, river, or air. This is in contrast to Guinea, Liberia, and Sierra Leone, where villages, towns, and capital cities are connected by an extensive network of footpaths, dirt roads, and paved highways. The West African 
road network is international: in the areas where Guinea, Liberia, and Sierra Leone have common borders, single ethnic groups inhabit more than one country, and cross-border travel is frequent, for purposes of commerce and to maintain family ties. Unlike the outbreak in the Boende area, the West African epidemic has been multinational almost from the start, with the transportation of both patients with EVD and bodies of deceased patients across borders, complicating the organization of public health measures.

The fourth possible explanation for the smaller outbreaks in equatorial Africa is that the response to Ebola outbreaks has been faster and more effective in the DRC and neighboring countries. With the experience of six previous EVD epidemics, the DRC is now well prepared. The time that it takes to respond to news of an outbreak has been shortened over the years. There is also substantial national expertise in managing EVD outbreaks, including skills in epidemiology, laboratory analysis, and patient care, with readily available international support. During the present outbreak, action was quickly taken to inform the affected communities and to assign responsibilities for control measures to village chiefs, religious and social leaders, traditional healers, and medical staff members. These actions were coupled with the introduction of basic infection-control measures - for example, on the basis of the slogan "no family without detergent," hand sanitizers were distributed to all affected communities to help stop transmission.

Finally, EVD may have spread more widely in West Africa because the human population is less resistant to infection than in equatorial Africa, either because there has been no previous exposure to EBOV or for some other reason. This possibility has not yet been investigated because, for example, we currently have little information about the risk that EVD will develop in a person after exposure to EBOV.

Although none of these possibilities can be ruled out, the third and fourth possibilities appear to be the most plausible on the basis of the current evidence. The coming days and weeks will reveal whether the new EBOV variant that was isolated in the Boende area is on the point of being eliminated from the human population, as in previous EVD outbreaks in the DRC. Regardless of the clinical and epidemiologic characteristics of this outbreak that are associated with the virus or the animal and human host populations, a continuing, comprehensive public health response will be critical for success.

\footnotetext{
The views expressed in this article are those of the authors and do not necessarily reflect the views of the national governments or the WHO.

Supported by Centre International de Recherches Médicales de Franceville (CIRMF), which is funded by the Gabonese government, Total Gabon, and the French Foreign Ministry; and by Metabiota, which is funded by the U.S. Department of Defense Armed Forces Health Surveillance Center, Division of Global Emerging Infections, Surveillance Operations (AFHSC GEIS), the Henry M. Jackson Foundation for the Advancement of Military Medicine, the Defense Threat Reduction Agency Cooperative Biological Engagement Program (DTRA-CBEP), Google.org, the Skoll Foundation, the U.S. Agency for International Development (USAID) Emerging Pandemic Threats Program, and the PREDICT project (cooperative agreement number GHN-A-OO-09-00010-00).

Disclosure forms provided by the authors are available with the full text of this article at NEJM.org.

We thank Philippe Engandja (CIRMF Gabon) and Valérie Caro and Laure Diancourt (Institut Pasteur de Paris) for their technical assistance; Ravi Santhana Gopala Krishna (WHO) and Hugh Keegan (ESRI [Environmental Systems Research Institute]) for providing the original version of the map in Figure 1; Jens $\mathrm{H}$. Kuhn for assistance in the preparation of the manuscript; and Joseph Fair for continuing support.
}

REFERENCES

1. Ebola virus disease: fact sheet no. 103 , updated September 2014. Geneva: World Health Organization (http://www.who.int/ mediacentre/factsheets/fs103/en/).

2. WHO Ebola Response Team. Ebola virus disease in West Africa - the first 9 months of the epidemic and forward projections. N Engl J Med 2014;371:1481-95. 3. Epelboin A, Formenty P, Anoko J, Allarangar Y. Humanisation and informed consent for people and populations during responses to VHF in central Africa (2003-2008). In: Biquet JM, ed. Humanitarian stakes. Geneva: MSF, 2008:25-37.

4. Towner JS, Sealy TK, Khristova ML, et al. Newly discovered Ebola virus associated with hemorrhagic fever out- break in Uganda. PLoS Pathog 2008;4(11): e1000212.

5. Berthet N, Reinhardt AK, Leclercq I, et al. Phi29 polymerase based random amplification of viral RNA as an alternative to random RT-PCR. BMC Mol Biol 2008;9:77.

6. Bankevich A, Nurk S, Antipov D, et al. SPAdes: a new genome assembly algorithm and its applications to single-cell sequencing. J Comput Biol 2012;19:455-77.

7. Tamura K, Peterson D, Peterson N, Stecher G, Nei M, Kumar S. MEGA5: molecular evolutionary genetics analysis using maximum likelihood, evolutionary distance, and maximum parsimony methods. Mol Biol Evol 2011;28:2731-9.
8. Ronquist F, Huelsenbeck JP. MrBayes 3: Bayesian phylogenetic inference under mixed models. Bioinformatics 2003;19: 1572-4.

9. Baize S, Pannetier D, Oestereich L, et al. Emergence of Zaire Ebola virus disease in Guinea. N Engl J Med 2014;371:141825.

10. Gire SK, Goba A, Andersen KG, et al. Genomic surveillance elucidates Ebola virus origin and transmission during the 2014 outbreak. Science 2014;345:136972.

11. Briand S, Bertherat E, Cox P, et al. The international Ebola emergency. $\mathrm{N}$ Engl J Med 2014;371:1180-3.

Copyright () 2014 Massachusetts Medical Society. 